\title{
Multilinguales
}

$14 \mid 2020$

Enseignement universitaire et professionnalisation : enjeux, questionnements et défis socio-économiques

\section{Les interrogatives dans le français parlé radiophonique algérien : approches sociolinguistique et socio-stylistique}

The interrogatives in spoken Algerian radio Franch : sociolinguistic and sociostylistic approaches

\section{Hamza Kernou}

\section{(2) OpenEdition}

1 Journals

\section{Édition électronique}

URL : https://journals.openedition.org/multilinguales/5695

DOI : 10.4000/multilinguales.5695

ISSN : 2335-1853

Éditeur

Université Abderrahmane Mira - Bejaia

\section{Référence électronique}

Hamza Kernou, «Les interrogatives dans le français parlé radiophonique algérien : approches sociolinguistique et socio-stylistique », Multilinguales [En ligne], 14 | 2020, mis en ligne le 10 décembre 2020, consulté le 02 juillet 2021. URL : http://journals.openedition.org/multilinguales/5695; DOI : https://doi.org/10.4000/multilinguales.5695

Ce document a été généré automatiquement le 2 juillet 2021. \footnotetext{
Pas d'Utilisation Commerciale - Pas de Modification 4.0 International
}

Multilinguales est mise à disposition selon les termes de la Licence Creative Commons Attribution - 


\section{Les interrogatives dans le français parlé radiophonique algérien : approches sociolinguistique et socio-stylistique}

The interrogatives in spoken Algerian radio Franch : sociolinguistic and socio-

stylistic approaches

Hamza Kernou

1 L'interrogation, qui constitue l'acte de langage le plus important pour la communauté parlante (Kerbrat-Orecchioni, 1991) est un domaine complexe. L'une de ses complexités réside dans la diversité des structures interrogatives, ainsi l'interrogation pose crucialement le problème de la signification à attribuer à la diversité des formes possibles (Gadet, 1989). En effet, plusieurs linguistes admettent la multiplicité des structures interrogatives dans le français parlé et ce depuis longtemps, car déjà en 1921 Foulet (1921) va jusqu'à dire que rien de plus varié que les formes de l'interrogation en français moderne. Cependant, une question se pose : comment expliquer cette diversité des structures interrogatives? Pour répondre à cette question, certains linguistes optent pour une approche grammaticale (Blanche-Benveniste, 1997), d'autres pour une approche pragmatique (Arrighi, 2007; Kernou et Sadi, 2018) et puis nous avons des linguistes qui optent pour une approche sociolinguistique, comme c'est le cas avec le présent travail, on peut citer (Barbarie, 1982 ; Coveney, 1997; Gadet, 1997 ; Quillard, 2001). De ce fait, l'interrogation dans le français parlé a fait l'objet de plusieurs approches.

Dans un article portant sur la variation des interrogatives Dagnac explique que :

La variation, qu'elle soit à base géographique, sociale, stylistique ou diaphasique, semble se traduire assez rarement de manière binaire (présence/absence d'une structure), mais bien plus souvent en terme de fréquence d'apparition des différentes structures, et son évaluation reste tributaire de la constitution des corpus et de leur mode d'exploitation. (Dagnac, $2013: 2$ ). 
Ceci nous permet de comprendre que l'approche sociolinguistique des interrogatives dans le français parlé porte sur la répartition des structures interrogatives selon les variables sociolinguistiques.

\section{Questionnements}

3 En partant du principe que les locuteurs appartenant à une même communauté linguistique n'ont pas forcément tous ni toujours les mêmes usages, nous nous intéresserons dans ce travail à l'étude de la variation sociolinguistique et sociostylistique des interrogatives dans un corpus de français parlé radiophonique algérien et dans des situations d'interactions et de contacts de langues, entre des animateurs ,des auditeurs, des invités et des invités assistants. Pour ce faire, nous avons choisi de prendre en considération une seule variable sociolinguistique et ce par souci de représentativité et surtout de faisabilité, mais aussi parce qu'il s'agit là d'un élément de variation très important, à savoir la variable « catégorie d'interactants ». En effet, notre corpus recèle beaucoup d'intervenants relevant de plusieurs profils sociolinguistiques. Il s'agira donc, dans ce présent travail, d'étudier la répartition de nos structures interrogatives partielles entre les trois catégories d'interactants (animateurs, auditeurs et médecins).

4 Nous avons pu comprendre jusqu'ici à quel point l'interrogation dans le français parlé représente un domaine riche mais surtout complexe. Dès lors, la problématique de la diversité des structures interrogatives s'impose et plusieurs questions nous viennent à l'esprit: Y a-t-il une variation sociolinguistique dans l'usage des interrogatives dans le français parlé radiophonique algérien? Cela nous conduit aussi à se poser les questions suivantes: Est-ce que la catégorie d'interactants constitue un facteur de variation sociolinguistique? Et quelles différences entre les différentes catégories?

\section{Corpus}

Pour répondre à ces différentes questions, nous avons choisi de travailler sur des données authentiques, un corpus constitué de trois émissions radiophoniques interactives d'Alger-Chaîne III, l'unique chaîne radiophonique d'expression française en Algérie, afin de disposer d'un discours en langue française, caractérisées par une diversité de thèmes, de cadre d'interaction et de type d'intervenants. Nous avons réuni quatre numéros de chaque émission. Ce qui constitue au total douze numéros.

\section{Présentation des émissions}

6 Nous avons en premier lieu L'antenne est à vous, une émission à thème social. Il s'agit là d'une émission ouverte aux auditeurs pour ouvrir leurs cœurs, parler de leurs espoirs et de leurs préoccupations. Elle représente ainsi, un espace d'expression et de partage pour les gens en détresse et qui passent par des moments difficiles, ayant des problèmes de cœur, des soucis professionnels, personnels, familiaux, etc.

En deuxième lieu, nous avons Stéthoscope, une émission à thème médical qui traite de tout ce qui se rapporte à la santé. Elle revient sur différentes pathologies, sur des problèmes de santé publique. Elle représente ainsi une heure de débat autour d'une maladie bien précise, et ceci en invitant sur le plateau, des médecins spécialistes de 
cette maladie qui à travers l'émission expliquent la maladie, ses causes, ses conséquences mais aussi les moyens de prévention.

En troisième et dernier lieu, nous avons Hier j'avais vingt ans, une émission qui traite de la musique. Il s'agit là d'une émission où les auditeurs, appellent pour demander une chanson qu'ils aimeraient écouter. Elle est aussi, une émission très riche en termes de conversation. Ce qui représente une heure de rire, d'humour, de nostalgie, de remémoration et de plaisir.

7 Nous avons effectué une transcription manuelle, orthographique et intégrale de la totalité des enregistrements (douze numéros). Cela fait un total de (208) pages, soit 69140 mots. L'antenne est à vous en contient 26767 mots sur (82) pages, Stéthoscope avec 24632 mots sur (69) pages et enfin Hier j'avais vingt ans en compte 17741 mots sur (57) pages.

\section{Présentation des interrogatives}

8 Nous avons repéré dans notre corpus (590) énoncés interrogatifs parmi lesquels on distingue (7) interrogatives inachevées qui appartiennent à la catégorie des interrogatives écartées, (30) interrogatives issues du contact de langues (français, arabe et berbère) et (553) interrogatives appartiennent au français. Cette dernière catégorie est composée de (13) interrogatives indirectes et de (540) interrogatives directes.

Parmi les interrogatives directes, nous avons (314) interrogatives totales qui se répartissent en seulement quatre structures interrogatives et (226) interrogatives partielles. Ces dernières, qui feront l'objet de cet article, sont les plus importantes pour notre étude car elles sont les plus riches en termes de diversité structurelle.

\section{Système de représentation adopté}

9 Il existe plusieurs systèmes de représentations pour décrire les différentes structures interrogatives partielles. Pour étudier notre corpus, nous adoptons un système de représentations, facile à lire et à comprendre, proposé par Coveney. Ce dernier dont les travaux sur l'interrogative sont nombreux, propose un système de représentations abrégées, celui-ci :

Peut sembler quelque peu simpliste du point de vue de théories syntaxiques formalistes, mais il a le mérite d'être facilement compréhensible par des chercheurs travaillant dans des approches très différentes. (Coveney, $2011: 215$ ).

Dans la liste suivante, l'exemple de chaque structure est précédé par sa représentation abrégée et un nom possible :

Tableau 1 : système de représentation des interrogatives partielles

\begin{tabular}{|l|l|l|}
\hline Structures interrogatives & Noms des structures & Exemples \\
\hline SVQ & In situ & Ils sont partis où? \\
\hline QSV & Antéposition & Où ils sont partis? \\
\hline QV-CL & Inversion du clitique & Où sont-ils partis? \\
\hline
\end{tabular}




\begin{tabular}{|l|l|l|}
\hline Q GNV-CL & Inversion complexe & Où les autres sont-ils partis? \\
\hline QVGN & Inversion stylistique & Où sont partis les autres? \\
\hline seQkSV & Clivage & C'est où qu'ils sont partis? \\
\hline QESV & 'Est-ce que' & Où est-ce qu'ils sont partis? \\
\hline QsekSV & 'variante d'est-ce que' & Où c'est qu'ils sont partis? \\
\hline QkSV & 'Complémenteur' & Où qu'ils sont partis? \\
\hline Q =SV & Sujet qu & Lesquels sont partis? \\
\hline
\end{tabular}

LÉgENDE : $S=$ sujet (clitique ou nominal) $; \mathrm{V}=$ verbe $; \mathrm{E}=$ est-ce que $; \mathrm{CL}=$ sujet clitique postposé $; \mathrm{GN}=$ Groupe Nominal ; $\mathrm{Q}=$ mot ou groupe interrogatif qu; $\mathrm{se}=c^{\prime} \mathrm{est}^{\prime} \mathrm{k}=$ que/qui ; ${ }^{\prime} \mathrm{Q}=\mathrm{S}^{\prime}=$ le sujet est un syntagme qu.

\section{Evaluation socio-stylistique des variantes de l'interrogation partielle}

Avant d'étudier la répartition des structures interrogatives partielles selon la catégorie des interactants, nous proposons d'effectuer dans ce qui suit une répartition des structures interrogatives partielles selon leur valeur socio-stylistique, c'est-à-dire selon le registre de langue auquel elles appartiennent et dans lequel elles sont produites. Et ceci en nous appuyant sur le tableau proposé par Coveney (2011).

En présentant un consensus concernant la valeur socio-stylistique accordée par les grammairiens à chacune des variantes interrogatives, Coveney affirme que les différentes structures peuvent être communément différenciées selon leur valeur socio-stylistique. Dans ce cas, nous prendrons la valeur socio-stylistique de la structure interrogative partielle comme l'un des critères de comparaison dans notre étude de la répartition des structures interrogatives partielles entre les différentes catégories d'interactants.

Tableau 2 : évaluation socio-stylistique des variantes de l'interrogation partielle

\begin{tabular}{|l|l|}
\hline Valeurs socio-stylistiques & Structures interrogatives \\
\hline Ecrit formel ; parler soutenu & Q V-CL \\
\hline Neutre & Q =S V, QV GN \\
\hline Neutre (mais parfois 'inélégant' à l'écrit) & QESV \\
\hline Familier (mais non stigmatisée à l'oral) & SVQ \\
\hline Hypercorrection & Q =S V-CL \\
\hline Familier/populaire & QSV, seQkSV \\
\hline
\end{tabular}


\begin{tabular}{|l|l|}
\hline Populaire & QkSV, QsekSV \\
\hline
\end{tabular}

11 Ce tableau représente, donc, la distribution socio-stylistique des structures (variantes) interrogatives selon un critère stylistique ( $\mathrm{du}$ plus soutenu au plus familier). En d'autres termes, la variation socio-stylistique concerne la répartition des unités linguistiques en registres, niveaux ou encore en styles de langue. On parle de trois registres de langue, nous avons le registre soutenu, le registre neutre et le registre familier/populaire.

Dans ce cas, nous constatons la présence de trois variétés socio-stylistiques de structures interrogatives partielles majeures. Nous avons deux variétés familières/ populaires (QSV et SVQ), deux variétés qui relèvent du parler neutre (QESV et QVGN) et une variété du parler soutenu (Q V-CL).

\section{Répartition des structures interrogatives partielles selon les catégories d'interactants}

L'étude de notre corpus nous a permis de relever la présence de vingt (20) structures interrogatives partielles. Parmi celles-ci, nous avons cinq (5) structures interrogatives partielles majeures qui comportent un nombre assez important d'interrogatives et quinze (15) structures interrogatives partielles mineures. Celles-ci sont très peu nombreuses dans notre corpus.

Le tableau suivant présente le nombre d'interrogatives pour les cinq structures interrogatives partielles majeures.

Tableau 3 : répartition des structures interrogatives partielles majeures

\begin{tabular}{|l|l|l|l|}
\hline $\mathrm{N}^{\circ}$ & Structures interrogatives & Nombre d'interrogatives & $\%$ \\
\hline 1 & {$[\mathrm{QSV}]$} & 51 & 22,57 \\
\hline 2 & {$[\mathrm{QV} \mathrm{GN}]$} & 42 & 18,58 \\
\hline 3 & {$[\mathrm{Q}$ V-CL $]$} & 35 & 15,49 \\
\hline 4 & {$[\mathrm{QESV}]$} & 31 & 13,72 \\
\hline 5 & {$[\mathrm{SVQ}]$} & 16 & 7,08 \\
\hline Autres structures & 51 & 22,57 \\
\hline \multicolumn{2}{|l|}{ Total } & 226 & $100 \%$ \\
\hline
\end{tabular}

Par souci de représentativité, notre étude va porter sur la répartition des cinq structures interrogatives partielles majeures selon la catégorie d'interactants, tout en prenant en considération leur valeur socio-stylistique et en se basant sur le modèle proposé par Coveney (2011). 
La catégorie d'interactants représente, selon nous, un facteur de variation sociolinguistique très important car notre corpus est constitué de plusieurs profils sociolinguistiques des locuteurs et de plusieurs catégories d'interactants. Nous avons relevé dans notre corpus la présence de (67) interactants différents. Ce chiffre inclut tous les participants de la conversation à savoir les animateurs, les auditeurs, les invités (médecins) et un invité assistant.

Notre corpus est composé de :

- Un animateur pour chacune des trois émissions (deux hommes et une femme). Nous retrouvons aussi dans le premier numéro de la deuxième émission un invité assistant, le seul de notre corpus. Ce dernier est un journaliste qui a participé dans cette émission au côté de l'animatrice habituelle. Cependant, ce journaliste ne sera pas pris en considération dans notre étude car il n'a formulé qu'une seule interrogative partielle ayant pour structure syntaxique [QGN], ce qui n'est pas représentatif. La voici :

- $I_{\mathrm{A}}$ : quelle relation entre les deux ? (E. 2, N. 1, P. 17)

- Dix invités dans les trois émissions dont 9 médecins dans la deuxième émission et un chanteur dans la troisième émission. Dans cette catégorie d'interactants, nous retenons les 9 médecins et ceci pour deux raisons: il s'agit là d'une catégorie d'interactants avec un nombre très représentatif et sont réputés pour leur maitrise de la langue française.

- Cinquante-trois auditeurs dans les trois émissions.

Le travail effectué ci-dessus nous permet donc de dégager trois catégories différentes d'interactants (les animateurs, les médecins et les auditeurs), dont nous étudierons la répartition des structures interrogatives partielles qu'ils produisent. Pour ce faire, nous dressons le tableau suivant :

Tableau 4 : répartition des structures interrogatives partielles selon les catégories d'interactants

\begin{tabular}{|l|l|l|l|l|l|l|}
\hline $\mathrm{N}^{\circ}$ & Structures interrogatives & Total & Animateurs & Médecins & Auditeurs & Exclues \\
\hline 1 & {$[\mathrm{QSV}]$} & 51 & 30 & 3 & 17 & 1 \\
\hline 2 & {$[\mathrm{QV} \mathrm{GN}]$} & 42 & 31 & 4 & 7 & 0 \\
\hline 3 & {$[\mathrm{Q}$ V-CL $]$} & 35 & 15 & 3 & 14 & 3 \\
\hline 4 & {$[\mathrm{QESV}]$} & 31 & 20 & 5 & 3 & 3 \\
\hline 5 & {$[\mathrm{SVQ}]$} & 16 & 9 & 2 & 5 & 0 \\
\hline Autres structures & 51 & 26 & 9 & 15 & 1 \\
\hline \multirow{2}{*}{ Total } & 226 & $57,96 \%$ & $11,5 \%$ & $26,99 \%$ & $3,54 \%$ \\
\cline { 4 - 8 } & $100 \%$ & 226 & & 61 & 8 \\
\hline
\end{tabular}

L'étude du tableau ci-dessus nous permet d'aboutir à plusieurs conclusions :

Nous constatons en premier lieu une dominance en matière de nombre d'interrogatives partielles produites par les animateurs, à savoir 131 interrogatives, ce qui représente 
57, $96 \%$ de la totalité des interrogatives partielles. Ceci pour la simple raison que, poser des questions est l'un des rôles essentiels de l'animateur de radio qui fait usage de questions pour faire durer, lancer ou relancer une conversation (Kernou et Sadi, 2018). En deuxième lieu, nous avons 61 interrogatives partielles produites par les auditeurs avec un pourcentage de 26, $99 \%$. En troisième lieu, nous avons la catégorie des médecins avec un nombre moins important, à savoir 26 interrogatives partielles, soit $11,5 \%$. En dernier lieu, nous avons 8 interrogatives partielles que nous avons décidé d'exclure de notre étude. Celles-ci sont produites par des interactants qui ne sont pas représentatifs en matière de nombre. Nous avons 7 interrogatives partielles produites par le seul invité de la troisième émission et une seule interrogative produite par l'unique invité assistant dans la deuxième émission.

Nous proposons le graphique suivant qui illustre toutes ces données:

Graphique 1 : répartition des interrogatives partielles entre les catégories d'interactants

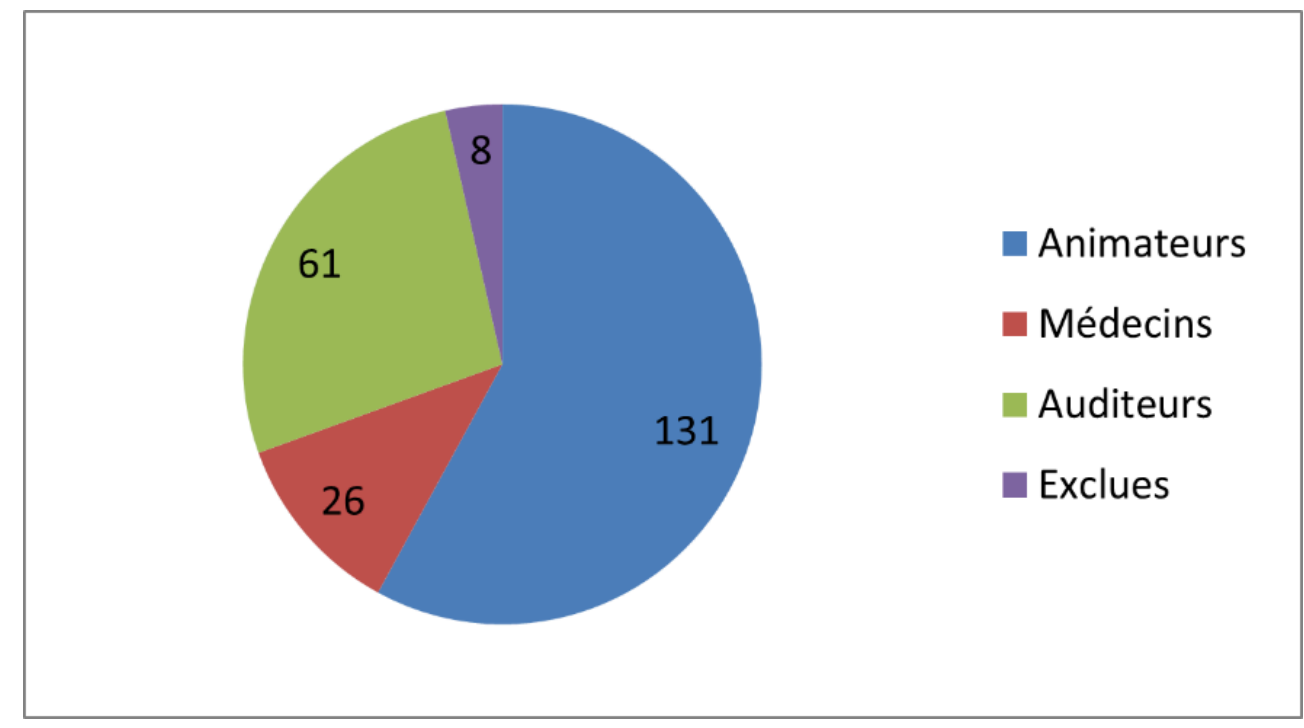

\section{Les animateurs}

Il s'agit là de la catégorie d'interactants qui a produit le plus grand nombre d'interrogatives partielles. Celles-ci, en nombre de 131, relèvent de 19 structures différentes dont 105 appartiennent aux 5 structures interrogatives partielles majeures. Nous dressons le tableau suivant qui synthétise les données de ces cinq structures.

Tableau 5 : les structures interrogatives partielles majeures chez les animateurs

\begin{tabular}{|l|l|l|l|}
\hline $\mathrm{N}^{\circ}$ & Structures interrogatives & Nombre & $\%$ \\
\hline 1 & {$[\mathrm{QV} \mathrm{GN}]$} & 31 & 29,52 \\
\hline 2 & {$[\mathrm{QSV}]$} & 30 & 28,57 \\
\hline 3 & {$[\mathrm{QESV}]$} & 20 & 19,05 \\
\hline 4 & {$[\mathrm{Q}$ V-CL $]$} & 15 & 14,29 \\
\hline
\end{tabular}




\begin{tabular}{|l|l|l|l|}
\hline 5 & {$[\mathrm{SVQ}]$} & 9 & 8,57 \\
\hline Total & 105 & $100 \%$ \\
\hline
\end{tabular}

L'observation du tableau ci-dessus nous permet d'aboutir à de nombreuses conclusions :

Premièrement, nous remarquons que les animateurs d'Alger-Chaîne III emploient un grand nombre d'interrogatives partielles avec une valeur socio-stylistique neutre. Celles-ci sont en nombre de 51 dans notre corpus. En effet, nous avons 31 interrogatives partielles en [QV GN] et 20 en [QESV]. Voici un exemple de chaque structure :

- [QV GN] A : quelle est la clé ? (E. 1, N. 1, P. 7)

- [QESV] A : qu'est-ce que vous en pensez ? (E. 2, N. 1, P. 12)

Deuxièmement, nous avons constaté la présence de 39 interrogatives partielles qui appartiennent au registre familier / populaire. Celles-ci sont produites par les animateurs avec la structure [QSV] (30 énoncés) et la structure [SVQ] (9 énoncés).

Il s'agit là d'un constat très important qui nécessite beaucoup d'explications. Cet usage important de ces variétés populaires par les animateurs (notamment ceux de la première et de la troisième émission), qui sont des professionnels de la radio ayant une grande maitrise de la langue française, nous fait comprendre qu'ils ne font pas toujours usage du parler soutenu et ceci pour plusieurs raisons. Ainsi, pour comprendre cet usage du parler populaire, il faut revenir au rôle et à la fonction d'animateur de la radio. En occupant ce poste, l'animateur s'adresse à des auditeurs mais surtout au grand public. En effet, pour se faire comprendre, l'animateur « qui dispose du pouvoir de donner la parole publique aux auditeurs » (Ravazzolo, $2007: 151$ ), adopte le parler de l'auditeur. Ceci permet aussi un meilleur déclenchement et déroulement de la conversation, d'où cet usage important du parler populaire.

Ainsi, cet usage par l'animateur du parler populaire, n'est nullement un signe d'incompétence linguistique, au contraire, il dénote surtout une compétence communicative. Cette compétence communicative permet à l'animateur de se familiariser avec l'auditeur, parler en familier pour se familiariser.

19 Troisièmement et dernièrement, nous avons constaté la présence de 15 énoncés interrogatifs produits par les animateurs dans une structure interrogative partielle qui appartient au parler soutenu, à savoir [Q V-CL]. Il s'agit là généralement des interrogatives qui connaissent un certain usage dans la société ${ }^{1}$. Voici un exemple :

-A : comment allez-vous ? (E. 1, N. 1, P. 2)

Bien que l'énoncé ci-dessus appartient au registre soutenu, il demeure d'un usage assez important dans la société.

20 Le travail effectué ci-dessus nous a permis de comprendre que les animateurs des émissions radiophoniques interactives d'Alger-Chaîne III varient, dans leurs usages des interrogatives partielles, entre les registres de langue avec une préférence pour le registre neutre, qu'on peut qualifier aussi de standard ainsi qu'un usage assez important du parler familier/populaire. 


\section{Les médecins}

Comme deuxième catégorie d'interactants, nous avons les médecins qui interviennent dans la deuxième émission. Ces derniers ont produit 26 interrogatives qui relèvent de 10 structures interrogatives partielles différentes.

Nous proposons le tableau suivant qui illustre l'usage, par les médecins, des différentes structures interrogatives partielles majeures :

Tableau 6 : les structures interrogatives partielles majeures chez les médecins

\begin{tabular}{|l|l|l|l|}
\hline $\mathrm{N}^{\circ}$ & Structures interrogatives & Nombre & $\%$ \\
\hline 1 & {$[\mathrm{QESV}]$} & 5 & 29,41 \\
\hline 2 & {$[\mathrm{QV} \mathrm{GN}]$} & 4 & 23,53 \\
\hline 3 & {$[\mathrm{Q}$ V-CL $]$} & 3 & 17,65 \\
\hline 4 & {$[\mathrm{QSV}]$} & 3 & 17,65 \\
\hline 5 & {$[\mathrm{SVQ}]$} & 2 & 11,76 \\
\hline Total & 17 & $100 \%$ \\
\hline
\end{tabular}

L'observation du tableau ci-haut nous permet de constater que les médecins ont fait usage, dans notre corpus, de 11 structures interrogatives partielles, un résultat semblable à celui obtenu avec les animateurs. En effet, nous dégageons aussi avec les médecins trois catégories socio-stylistiques d'interrogatives partielles.

En premier lieu, nous avons 9 interrogatives partielles produites par les médecins appartenant au registre neutre. Nous comptons 5 exemples avec la structure [QESV] et 4 exemples avec la structure [QV GN].

Voici dans ce qui suit un exemple de chaque structure :

- [QESV] $\mathrm{I}_{1}$ : qu'est-ce qu'on fait du conseil génétique ? (E. 2, N. 3, P. 15)

- [QV GN $]_{3}$ : quels sont les effets secondaires ? (E. 2, N. 4, P. 3)

En deuxième lieu, nous constatons que les médecins ont fait usage, dans notre corpus, de 5 énoncés interrogatifs partiels, qui relèvent du registre familier / populaire. Il s'agit des structures [QSV] et [SVQ] avec 3 énoncés interrogatifs pour la première et 2 pour la seconde.

Considérons dans ce qui suit, un exemple de chaque structure :

- [QSV] $\mathrm{I}_{3}$ : de quoi il s'agit? (E. 2, N. 4, P. 3)

- [SVQ] $\mathrm{I}_{2}$ : bilan hépatique perturbé mais pourquoi ? (E. 2, N. 3, P. 5)

En dernier lieu, nous avons trois interrogatives partielles utilisées par les médecins et qui appartiennent au registre soutenu. Celles-ci sont formées dans la structure [Q V$\mathrm{CL}$.

Voici un exemple :

-[Q V-CL] $\mathrm{I}_{1}$ : comment ce fait-il ? (E. 2, N. 3, P. 9) 
L'étude menée ci-dessus nous a permis de constater que les médecins, comme les animateurs, favorisent beaucoup plus le recours aux interrogatives d'un registre neutre avec un usage assez important d'interrogatives appartenant au registre dit familier et/ ou populaire ainsi que quelques interrogatives issues du registre soutenu. Ainsi, les médecins, comme les animateurs, alternent entre les différents registres dans leur usage des interrogatives.

Pour comprendre les résultats obtenus ci-haut, il faut revenir au rôle des intervenants dans cette deuxième émission à thème médical. En effet, les médecins sont invités sur le plateau de l'émission pour discuter, débattre et expliquer une maladie bien précise.

Etant donné qu'il s'agit d'une émission radiophonique destinée au grand public, les médecins qui interviennent dans cette émission tentent de faire usage d'un parler accessible au grand public. On parle dans ce cas de vulgarisation scientifique.

Ainsi, le rôle et la fonction première de cette émission radiophonique interactive est de vulgariser le savoir scientifique et plus particulièrement le savoir médical. Ceci, pour informer, conseiller et sensibiliser le grand public.

En effet, en dehors des termes de spécialités, les médecins qui interviennent dans cette émission font usage d'une langue très accessible au grand public. Ceci expliquerait donc cette alternance de registres de langue dans les interrogatives partielles.

\section{Les auditeurs}

Comme troisième et dernière catégorie d'interactants, nous avons les auditeurs des trois émissions. Ceux-ci, en nombre de 53, ont formulés 61 interrogatives partielles qui sont produites dans 11 structures différentes, dont 46 appartiennent aux cinq structures interrogatives partielles majeures.

En suivant le même principe qu'avec les deux autres catégories d'interactants, nous présenterons dans ce qui suit un tableau qui synthétise le nombre d'interrogatives partielles produites dans chaque structure interrogative partielle majeure.

Tableau 7 : les structures interrogatives partielles majeures chez les auditeurs

\begin{tabular}{|l|l|l|l|}
\hline $\mathrm{N}^{\circ}$ & Structures interrogatives & Nombre & $\%$ \\
\hline 1 & {$[\mathrm{QSV}]$} & 17 & 36,96 \\
\hline 2 & {$[\mathrm{Q}$ V-CL $]$} & 14 & 30,43 \\
\hline 3 & {$[\mathrm{QV} \mathrm{GN}]$} & 7 & 15,22 \\
\hline 4 & {$[\mathrm{SVQ}]$} & 5 & 10,87 \\
\hline 5 & {$[\mathrm{QESV}]$} & 3 & 6,52 \\
\hline Total & 46 & $100 \%$ \\
\hline
\end{tabular}

L'étude des interrogatives partielles produites par les auditeurs nous permet aussi de relever la présence de plusieurs interrogatives qui appartiennent aux cinq structures interrogatives partielles majeures. 
Sur le plan socio-stylistique, nous avons distingué aussi trois types de structures interrogatives. Cependant, les résultats obtenus avec cette troisième catégorie d'interactants diffèrent des résultats obtenus avec les deux autres catégories.

En première position, nous avons 22 énoncés interrogatifs partiels, produits par les auditeurs et qui appartiennent au registre familier/ populaire. Ces énoncés appartiennent aux deux structures syntaxiques suivantes : 17 énoncés avec la structure [QSV] et 5 énoncés avec la structure [SVQ].

Notons un exemple pour chaque structure :

- [QSV] $\mathrm{A}_{\mathrm{U} 2}$ : comment vous allez ? (E. 3, N. 4, P. 7)

-[SVQ] $\mathrm{A}_{\mathrm{U} 2}$ : tu as fais quoi ? (E. 1, N. 3, P. 14)

Ainsi, le registre familier et/ou populaire occupe la première place dans les interrogatives partielles produites par les auditeurs. Ceci n'est pas le cas des animateurs et des médecins où nous avions constaté que le registre neutre occupait la première place dans leurs interrogatives partielles.

30 En deuxième position, nous avons 14 interrogatives partielles produites par les auditeurs dans un registre soutenu avec la structure [Q V-CL]. Ces interrogatives apparaissent essentiellement dans la séquence d'ouverture de la conversation et dans certains énoncés interrogatifs qui connaissent un grand usage par les auditeurs. En témoignent les trois extraits suivants :

- $\mathrm{A}_{\mathrm{U} 1}$ : comment allez-vous? (E. 3, N. 4, P. 2)

- $\mathrm{A}_{\mathrm{U} 4}$ : qu'en est-il de ce type de soulagement maintenant ? (E. 2, N. 2, P. 9)

- $\mathrm{A}_{\mathrm{U} 2}$ : comment dirai-je ? (E. 1, N. 1, P. 11)

31 En troisième et dernière position, nous avons 10 interrogatives produites par les auditeurs et qui relèvent du registre neutre.

Nous en avons 7 avec la structure [QV GN] et 3 avec la structure [QESV]. Voici un exemple de chaque structure :

- $\left[\mathrm{QV}\right.$ GN] $\mathrm{A}_{\mathrm{U} 2}$ : pourquoi parler sur les autres? (E. 1, N. 3, P. 12)

- [QESV] $\mathrm{A}_{\mathrm{U} 1}$ : qu'est-ce que vous en pensez vous aussi ? (E. 1, N. 2, P. 3)

L'étude des interrogatives partielles produites par les auditeurs nous a permis d'aboutir à des résultats différents de ceux obtenus avec les animateurs et les médecins. L'alternance des registres existe toujours mais l'enchainement est différent.

Nous avons constaté avec cette troisième catégorie d'interactants, comparée aux deux autres catégories, un usage très important des interrogatives appartenant au registre familier. La grande différence entre ces deux catégories d'interactants se situe au niveau du registre neutre qui occupe la première place avec les animateurs et les médecins et la dernière place avec les auditeurs.

Nous avons étudié dans ce travail, consacré à une interprétation sociolinguistique et socio-stylistique de la diversité des structures interrogatives partielles, la répartition des structures interrogatives partielles selon la catégorie d'interactants où nous avons dégagé trois catégories de locuteurs (les animateurs, les médecins et les auditeurs).

Cette étude, nous a permis de confirmer notre hypothèse: "la catégorie d'interactants » représente un facteur de variation sociolinguistique.

Nous avons constaté une alternance de registres de langue pour toutes les catégories d'interactants. Les animateurs et les médecins favorisent beaucoup plus le recours aux interrogatives d'un registre neutre, appartenant au vocabulaire usuel, utilisé au quotidien dans la plupart des échanges, avec un usage assez important d'interrogatives 
appartenant au registre dit familier et/ou populaire ainsi que quelques interrogatives issues du registre soutenu. Nous avons constaté avec les auditeurs un usage très important des interrogatives appartenant au registre familier ainsi qu'un usage moins important des interrogatives du registre soutenu. Ainsi, cette interprétation sociolinguistique et socio-stylistique des interrogatives partielles nous a permis de mieux comprendre la diversité des structures interrogatives partielles. Cette étude nous a permis de constater la présence de plusieurs facteurs sociolinguistiques qui régissent la diversité des structures interrogatives partielles. Dans ce cas, la diversité structurelle des interrogatives partielles est à la fois due, sur le plan sociolinguistique, à la diversité des profils sociolinguistiques et socioprofessionnels des interactants, à la diversité des émissions ainsi qu'à leur finalité.

\section{BIBLIOGRAPHIE}

ARRIGHI, L., «L'interrogation dans un corpus de français parlé en Acadie. Formes de la question et visées de l'interrogation », Linx, 57, Paris, 2007, pp. 47-56.

BARBARIE, Y., « Analyse sociolinguistique de la syntaxe de l'interrogation en français québécois », Revue québécoise de linguistique, 121, Québec, 1982, pp. 145-167.

BEAUD, L et al., « ALORS ? COMMENT ALLEZ-VOUS ? Entrée en matière dans une consultation médicale », Langage et société, 126, Paris, 2008, pp. 57-74.

BLANCHE-BENVENISTE, C., « A propos de qu'est-ce que c'est et c'est quoi », Recherches sur le français parlé, 14, Aix-en-Provence, 1997, pp. 127-146.

COVENEY, A., « L'approche variationniste et la description de la grammaire du français : le cas des interrogatives », Langue française, 115, Paris, 1997, pp. 88-100.

COVENEY, A., « L'interrogation directe », Travaux de linguistique, 63, Liège, 2011, pp. 112-145.

DAGNAC, A., « La variation des interrogatives en français », document préparatoire (texte provisoire) pour contribution à la GGF (Abeille, A., Godard, G. et A., 2013. <hal-00988751>

FOULET, L., « Comment ont évolué les formes de l'interrogation », Romania, 47, Paris, 1921, pp. 243-348.

GADET, F., Le français ordinaire, Armand Colin, Paris, 1989.

GADET, F., « La variation de tous les français », Linx, 57, Paris, 1997, pp. 155-164.

KERBRAT-ORECCHIONI, C., La question, PUL, Lyon, 1991.

KERNOU, H et SADI, N., « La question dans le discours radiophonique algérien : quelle fonction pragmatique et interactive ? », Studii de gramatică contrastivă, 29, Roumanie, 2018, pp. 33-45.

QUILLARD, V., « La diversité des formes interrogatives : comment l'interpréter ? ", Langage et société, 95, Paris, 2001, pp. 57-72. 


\section{NOTES}

1. Nous renvoyons à l'article, paru en 2008, de Beaud, Cahagne et Guyard et dont le titre est très révélateur : «Alors ? Comment allez-vous?».

\section{RÉSUMÉS}

Ce travail a pour objectif d'effectuer une interprétation sociolinguistique et socio-stylistique de la diversité des structures interrogatives partielles dans le français parlé d'Alger-Chaîne III et ceci dans trois émissions radiophoniques interactives à thèmes social, médical et musical. Cette étude a montré des usages différents des interrogatives partielles selon les trois catégories d'interactants, animateurs, auditeurs et invités.

The aim of this work is to carry out a sociolinguistic and socio-stylistic interpretation of the diversity of partial interrogative structures in the spoken French of Algiers-Chain III and this in three interactive radio programs on social, medical and musical themes. This study shows different uses of partial interrogations according to the three categories of interactants, presenters, listeners and guests.

\section{INDEX}

Keywords : interrogative, radio speech, interaction, socio-stylistics, Algeria

Mots-clés : interrogatives, discours radiophonique, interaction, socio-stylistique, Algérie

\section{AUTEUR}

\section{HAMZA KERNOU}

Université de Bejaia, Laboratoire LESMS, Algérie 